\title{
Should Collision Avoidance Systems use Yaw Stabilization?
}

\author{
Daniel Heß, Matthias Althoff, Thomas Sattel
}

\begin{abstract}
Due to historical reasons or system development aspects, many high-level control tasks in vehicles are performed by underlying low-level controllers. This separation of concerns provides reliable systems, but potentially degrades the performance compared to centralized control. Performance losses are acceptable for most control tasks, but for collision avoidance systems one should not compromise on safety. We investigate the performance loss for collision avoidance systems when an underlying yaw stabilization controller is used, which can be found in many modern vehicles under various product names, such as electronic stability control (ESC). Since electronic stability control differs from vehicle to vehicle, we use an idealized controller that performs better than or equally well as an actual realization. It is shown that central control concepts bypassing the yaw stabilization perform better than a hypothetical controller embedded with the idealized yaw stabilization. We also provide a measure for the performance loss, which should support the decision for or against the use of yaw stabilization in collision avoidance systems.
\end{abstract}

\section{INTRODUCTION}

Comparing the performance of a vehicle with electronic stability control to a vehicle with a centralized control is a challenging task. The problem is that due to protection of intellectual property, makers of such systems do not reveal their control algorithms. Besides this issue, one would not only have to test different suppliers, but also different vehicles since electronic stability control is tuned for each vehicle differently, even regional customer preferences might be considered. In order to overcome those difficulties, we give electronic stability control the best chance: We compute the optimal open-loop control performance when the system does not suffer from sensor noise and when no tracking errors occur. This procedure has the advantage that we do not have to know details of any specific ESC algorithm. The performance degradation only origins from the constraint that a certain yaw rate has to be maintained, which causes a higher utilization of tire forces than in the unconstrained case. The nominal performance of tracking with ESC is compared to trajectory tracking with two centralized controllers. The first centralized controller employs a conventional constant brake balance, whereas the second one uses individual wheel braking and choses an optimal brake force distribution for trajectory tracking. The differences between yaw rate constrained performance of an ESC based control approach and the unconstrained performance of the centralized tracking controllers are put into perspective by

M. Althoff is with the department of Computer Science and Automation, D. Heß and T. Sattel are with the department of Mechanical Engineering, Ilmenau University of Technology, 98693 Ilmenau, Germany. \{Daniel.Hess, Matthias.Althoff, Thomas. Sattel \}eTU-Ilmenau.de considering the (finite horizon) optimal trajectory tracking solution. For the comparison we use a benchmark scenario proposed in an earlier work of the authors [6].

To the best knowledge of the authors, there are no contributions in the literature that are concerned with the coexistence of trajectory tracking in automated vehicle operation and electronic stability controllers. Previous results either solely focus on electronic stability control for humandriver assistance or on controllers for automated operation. Yaw stabilization concepts using individual braking torques are presented in [3], [17]. Optimization of electronic stability control by not only using negative brake torques, but also positive torque from torque vectoring can be found in [8], [12]. Concepts for yaw control allocation strategies including active steering are described in [2], [16], [19]. There are also many research activities for simultaneous yaw stabilization and suspension performance optimization [5], [14], [15].

For automated vehicle operation, we focus the literature research on contributions that use exact input-output linearization since this is the concept applied in this work. In [18] the steering angle and the overall braking force are used as system inputs. Similarly, in [7], [11] the steering angle and individual front- and rear-axle braking forces are used as system inputs. Controlling the individual brake discs is proposed in [10], yet with the downside that required inputs include steering angles for all wheels as well as active suspension. Trajectory tracking with the inputs steering angle and individual wheel braking has been considered in [4] in a model predictive control framework, although with the downside that exact tracking can not be guaranteed even in the nominal, disturbance free case.

In Sec. II the mathematical model of the vehicle is presented. The cost function for comparing the control concepts and optimal open-loop solutions are shown in Sec. III. Two proposed closed-loop controllers based on an exact inputoutput linearization for comparison with the idealized openloop control are described in Sec. IV: The first variant uses the angular velocity of the steering and a statically balanced brake-torque as system input. The second variant uses the angular velocity of the steering and individual brake torques. The results of the comparisons are summarized in Sec. V.

\section{VEHICLE MODEL}

A planar four-wheel vehicle model with a simplified suspension system and a nonlinear Paceijka-like tire model [10] is used. The state of the vehicle main body with the total mass $m$ and the rotational inertia $I_{z}$ is described by the velocity in longitudinal and lateral vehicle-relative direction $v_{x}^{C G}, v_{y}^{C G}$ of the center of gravity $(C G)$ and the rotational yaw 
velocity $\dot{\varphi}_{z}$. The wheels numbered one to four in the order front-left, front-right, rear-left and rear-right are positioned at $\rho_{i}$ measured relative to the vehicle center of gravity. For instance $\rho_{1}=\left(l_{f}, b,-h\right)^{T}$ for the front-left wheel $i=1$. We use the notation $a_{\{x, y\}}$ to denote a vector containing the $x$ and $y$ components of $a$. A wheel $i$ produces forces acting in vehicle $x, y$ and $z$ direction, $F_{i,\{x, y, z\}}$. The resulting vehicle equation of motion is affine in the inputs:

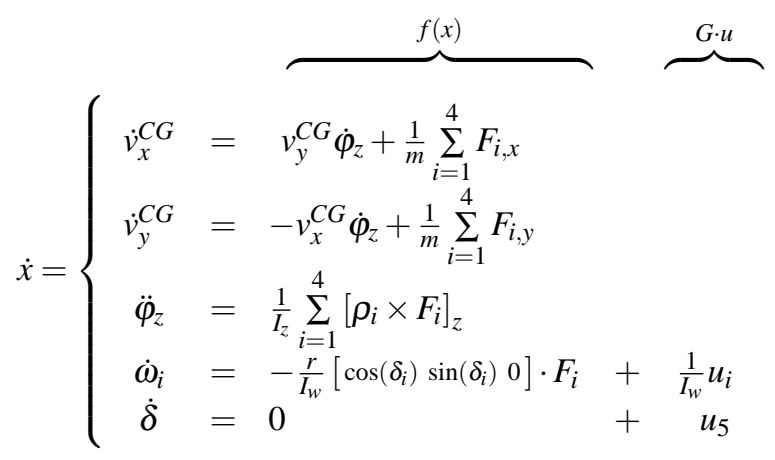

For each wheel $i=1 \ldots 4$ with radius $r$ and rotational inertia $I_{w}$ we have one differential equation for $\dot{\omega}_{i}$ in (1), leading to an eighth order system. The steering angle of the front wheels is set to $\delta_{1}=\delta_{2}=\delta$ and of the rear wheels $\delta_{3}=$ $\delta_{4}=0$. In following equations we use the rotation matrix

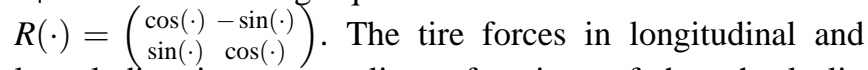
lateral direction are nonlinear functions of the wheel slip vector $s_{i}=\left(s_{i, x}, s_{i, y}\right)$, the steering angle $\delta_{i}$, and the wheel normal force $F_{i, z}$ :

$$
\begin{aligned}
& F_{i,\{x, y\}}=\mu_{0} \cdot F_{i, z} \cdot R\left(\delta_{i}\right) \mu\left(s_{i,\{x, y\}}\right) \\
& \mu(s)=-\frac{s}{\|s\|} \cdot \sin \left(C \arctan \left(B / \mu_{0} \cdot\|s\|\right)\right) \\
& s_{i,\{x, y\}}=\left(R\left(-\delta_{i}\right) v_{i}-\left(\begin{array}{rr}
r \omega_{i} & 0
\end{array}\right)^{T}\right) /\left\|v_{i}\right\| \\
& v_{i}=v_{\{x, y\}}^{C G}+\rho_{i} \times\left(\begin{array}{ccc}
0 & 0 & \dot{\varphi}_{z}
\end{array}\right)^{T}
\end{aligned}
$$

The simplified suspension is modeled under the assumption that roll and pitch angles are zero, $\varphi_{x} \equiv \varphi_{y} \equiv 0$, leading to the equilibrium condition:

$$
0=\sum_{i=1}^{4}\left[\rho_{i} \times F_{i}\right]_{\{x, y\}}, \quad m g=\sum_{i=1}^{4} F_{i, z}
$$

The resulting normal forces $F_{i, z}$ equal the steady state normal forces of a dynamic suspension system for small pitch and roll angles. The normal forces vary with different longitudinal and lateral vehicle accelerations, leading to higher load on the outer wheels in a curve and higher load on the front wheels for braking. The equilibrium is over-constrained for four wheels, which is resolved here by assuming that the front and the rear wheel of one side contribute equally to the roll equation. Equation (6) can be put in explicit form for $F_{i, z}$, if (2) depends linearly or quadratically on $F_{i, z}$.

\section{OPTIMAL TRAJECTORY TRACKING}

We first introduce in Sec. III-A the general optimization problem for unconstrained trajectory tracking. In Sec. III-B we add further constraints to consider the effects of an embedded electric stability control.

\section{A. Optimal Tracking without ESC}

We assume that a desired trajectory is given for the vehicle $C G$. The goal of this section is to produce a vehicle movement which is exactly constrained to the desired trajectory and which is optimal in the remaining degrees of freedom. At all points of time the length and the orientation of the CG's velocity vector has to equal the length $V(t)$ and the orientation $\theta(t)$ of the desired trajectory's velocity vector. A derivative with respect to time yields two constraints for exact tracking:

$$
\left(\begin{array}{c}
\dot{v}_{x}^{C G}-v_{y}^{C G} \dot{\varphi}_{z} \\
\dot{v}_{y}^{C G}+v_{x}^{C G} \dot{\varphi}_{z}
\end{array}\right)=R\left(\theta-\varphi_{z}\right)\left(\begin{array}{c}
\dot{V} \\
V \dot{\theta}
\end{array}\right)
$$

The objective function is defined similarly to [9] as the integral over the sum of the squared tire force transmission ratio:

$$
J=\int_{t_{0}}^{t_{f}} \frac{1}{4} \sum_{i=1}^{4}\left(\frac{\left\|F_{\{x, y\}, i}(t)\right\|}{\mu_{0} F_{z, i}(t)}\right)^{2} \mathrm{~d} t
$$

The local cost $\frac{d}{d t} J(t)$ is bounded by the interval $[0,1]$, where the value 1 indicates that the maximum transmissible tire force is used.

After defining the side slip angle $\beta=\theta-\varphi_{z}$ we can formulate the state vector of the dynamic optimization problem:

$$
x_{I}(t)=\left(\beta(t), \dot{\beta}(t), \ddot{\beta}(t), \omega_{1}(t), \ldots \omega_{4}(t), \delta(t)\right)^{T}
$$

with the initial conditions $\beta\left(t_{0}\right)=0$ and $\dot{\beta}\left(t_{0}\right)=0$. It is not necessary to solve for the system inputs as they are absent from the objective function and the constraints. We employ a local collocation approach [1] to transform the dynamic optimization problem to a static nonlinear optimization problem. The solution trajectory $x_{I}(t)$ is represented by $k$ orthogonal polynomials of degree $p$, that is $k(p+1)$ supports. The static optimization is solved by the Matlab ${ }^{\circledR}$ function fmincon.

\section{B. Optimal Tracking with ESC}

According to [13] the vehicle yaw dynamics can be stabilized in a way satisfying user expectancies by choosing the steady state rotational velocity $\dot{\varphi}_{0}(\boldsymbol{\delta})$ of the linear bicycle model:

$$
\dot{\varphi}_{0}(\delta)=\delta H(v)=\delta \frac{v}{l_{f}+l_{r}+m v^{2} \frac{l_{f} c_{f}-l_{r} c_{r}}{2\left(l_{f}+l_{r}\right) c_{f} c_{r}}}
$$

As the linear bicycle model does not describe the saturation of the tire forces, the desired value is additionally limited by a maximum lateral acceleration and its corresponding maximum rotational veloctiy:

$$
\dot{\varphi}_{z}^{*}=\max \left(-\frac{\mu g}{v}, \min \left(\delta \cdot H(v),+\frac{\mu g}{v}\right)\right)
$$

There are unknowns regarding ESC parametrization that require several assumptions: The activation threshold of a 
specific ESC system is not known. We therefore assume that the ESC is operational in the complete time interval of the investigated maneuver. The parameters of the feedback loop are also unknown. We assume that in reality $\dot{\varphi}_{z}$ never diverges far from the desired value $\dot{\varphi}_{z}^{*}$, so that the transient ESC behavior (settle-time etc.) and regulatory system inputs in comparison to nominal inputs are negligible. For the idealized yaw-control the constraint $\dot{\varphi}_{z} \equiv \dot{\varphi}_{z}^{*}$ is enforced. Another unknown is the method of allocating the required yaw moment to the brake inputs of the four wheels. We assume here that real ESC implementation uses a very good allocation method. The optimization problem is therefore simultaneously solved for the wheel-speeds as in the above, unconstrained case to yield an optimal allocation.

In total, we model trajectory tracking with active ESC as an optimization problem similar to III-A, but with the following constraints in addition to the free optimization problem:

$$
\begin{aligned}
& -\frac{\mu g}{V(t)} \leq \dot{\theta}-\dot{\beta} \leq \frac{\mu g}{V(t)} \\
& \ddot{\theta}-\ddot{\beta}=\dot{\delta}(t) H(V(t))+\delta(t) \frac{\partial H}{\partial V} V(t)
\end{aligned}
$$

These three constraints directly result from the ESC desired yaw rate $\dot{\varphi}_{z}^{*}$ given in (11), with the constraint (13) as the derivative of (10) being equivalent to (10) when the initial state satisfies (10).

\section{REACTIVE TRAJECTORY TRACKING}

One of the advantages of input-output linearization over other control methods is that it facilitates exact open-loop tracking under nominal conditions, (no measurement noise, process disturbances or model errors). This property is required for the comparison in Sec. V, as tracking-errors (cutting the corner) would lead to lower tire-force utilization and therefore distort the comparison. This section describes two trajectory tracking controllers based on input-output linearization. The first controller uses a conventional constant brake balance and the second actuates individual wheel brakes.

We start by defining a feedback that linearizes the velocity vector of a look-ahead position $P$. Position tracking can then be achieved by finding a suitable derivative of the tracking error.

The velocity vector of a vehicle-fixed point $P$ with the vehicle-relative position $\rho, \rho_{x} \geq 0$ is the quantity to be controlled:

$$
h_{P}:=v_{\{x, y\}}^{C G}+[\rho \times \dot{\varphi}]_{\{x, y\}}
$$

Using the braking torques $u_{1}=T_{1}, \ldots, u_{4}=T_{4}$ and the steering angle velocity $\dot{\delta}$ as inputs, one can show that $h_{P}$ has relative degree two for a reasonable set of vehicle states:

$$
\frac{\partial h_{P}}{\partial x} G=0, \quad \operatorname{rank}\left(\frac{\partial h_{P}}{\partial x} \frac{\partial f}{\partial x} G\right)=2
$$

Accordingly, we can directly manipulate the second derivative of the velocity of $P$ and a linearizing input vector $u$ therefore has to comply to:

$$
\begin{aligned}
h_{P}^{(2)} & =\frac{\partial h_{P}}{\partial x} \frac{\partial f}{\partial x} f(x)+\frac{\partial h_{P}}{\partial x} \frac{\partial f}{\partial x} G u \\
& =: a(x)+B(x) u
\end{aligned}
$$

The vector $\partial h_{P} / \partial x$ is constant and $\partial f / \partial x$ is easily computable by taking the numerical derivative of $f$ at the current state $x(t)$.

Trajectory tracking can be formulated as proposed in [18] by defining the path-tangential tracking error $\varepsilon_{T}$ and the path-normal error $\varepsilon_{N}$ with $\varepsilon=\left(\varepsilon_{T}, \varepsilon_{N}\right)^{T}$ as the difference between the actual look-ahead position $\left(X^{P}, Y^{P}\right)$ and the desired position $\left(X^{*}, Y^{*}\right)$ :

$$
\varepsilon=R(-\theta)\left(\begin{array}{c}
X^{P}-X^{*} \\
Y^{P}-Y^{*}
\end{array}\right)
$$

The desired trajectory can be specified in multiple ways. Here, we use the tangential (absolute) velocity $V(t)$ and the current heading $\theta(t)$, which is the angle between an earth-fixed coordinate system and the current tangent of the trajectory. With the directly controllable output derivative $h_{P}^{(2)}$ appearing in the third derivative $\varepsilon^{(3)}$ of the tracking error, the necessary velocity changes are:

$$
\begin{aligned}
h_{P}^{(2)} & =R\left(\theta-\varphi_{z}\right)\left(\varepsilon^{(3)}+\dddot{\theta} \bar{\varepsilon}+2 \ddot{\theta} \bar{\varepsilon}^{(1)}+\dot{\theta} \bar{\varepsilon}^{(2)}+\left(\begin{array}{c}
\ddot{V} \\
0
\end{array}\right)\right) \\
& -\left(\varphi_{z}^{(2)}-\ddot{\theta}\right) \bar{h}_{P}-2\left(\varphi_{z}^{(1)}-\dot{\theta}\right) \bar{h}_{P}^{(1)} \\
& +\left(\varphi_{z}^{(1)}-\dot{\theta}\right)^{2} h_{P},
\end{aligned}
$$

using the convenient notation $\bar{a}_{\{x, y\}}=\left(-a_{y}, a_{x}\right)^{T}$ to denote a vector rotated by 90 degrees. The error $\varepsilon^{(3)}$ may be used to define a feedback compensation.

\section{A. Tracking Controller with Static Brake Balance (SBB)}

To model trajectory tracking with conventional, nondifferential braking, the five system inputs individual braking torques and steering angle velocity, $u_{1 . . .5}$, are mapped to the two virtual inputs $v_{1}$ and $v_{2}$ with the help of the static brake balance $b_{b} \in[0,1]$ :

$$
u:=\left(\begin{array}{ccccc}
b_{b} & b_{b} & 1-b_{b} & 1-b_{b} & 0 \\
0 & 0 & 0 & 0 & 1
\end{array}\right)^{T} v
$$

After inserting (20) in (16), the equation can be solved for $v$ and then $u$. The resulting controller always distributes braking torques according to a static brake balance and thus models vehicle systems where individual wheel brakes are not accessible.

\section{B. Tracking Controller with Individual Wheel Braking (IWB)}

In case the trajectory tracking controller has access to individual brakes, it may actuate five inputs to satisfy the two equality constraints of (16). The three remaining degrees 
of freedom can be employed to minimize the following cost function:

$$
j_{u}:=u^{T} K u+c u
$$

One possibility is to chose $K$ so as to weigh the inputs by their maximum value,

$$
K_{i, i}:=\frac{1}{u_{i, \max }(x)}, \quad K_{i, j}=0, \text { for } i \neq j
$$

with

$$
u_{i, \max }(x):=r \cdot \sqrt{\mu_{0}^{2} F_{z, i}^{2}-F_{y, i}^{2}}, \quad 1 \leq i \leq 4,
$$

assuming a nearly isotropic tire model, and

$$
u_{5, \max }:=\dot{\delta}_{\max },
$$

because the steering angle velocity is limited by the physical properties of the steering motor. A cost function $j_{x}$ depending on the vehicle state can be locally minimized in a gradient descent fashion by minimizing the time-derivative $\frac{d}{d t} j_{x}=$ $\frac{\partial j_{x}}{\partial x}(f(x)+G u)$. If we select $j_{x}=\frac{1}{2} \delta^{2}$ with $\frac{d}{d t} j(x)=\delta \dot{\delta}$, we can set

$$
c(x):=(0,0,0,0, \delta) .
$$

To minimize the cost function $j_{u}$, while at the same time satisfying the path tracking constraints (16), the following Lagrange function is used:

\begin{tabular}{|c|c|c|c|c|c|}
\hline \multicolumn{3}{|c|}{ VEHICLE PARAMETERS } & \multirow[b]{2}{*}{$\begin{array}{l}l_{r}[\mathrm{~m}] \\
1.27\end{array}$} & \multirow[b]{2}{*}{$\begin{array}{l}h[\mathrm{~m}] \\
0.5\end{array}$} & \multirow[b]{2}{*}{$\begin{array}{l}b[\mathrm{~m}] \\
0.74\end{array}$} \\
\hline $\begin{array}{l}m[\mathrm{~kg}] \\
1750\end{array}$ & $\begin{array}{l}J_{z}\left[\mathrm{~kg} \mathrm{~m}^{2}\right] \\
2500\end{array}$ & $\begin{array}{l}l_{f}[\mathrm{~m}] \\
1.43\end{array}$ & & & \\
\hline $\begin{array}{l}r[\mathrm{~m}] \\
0.32\end{array}$ & $\begin{array}{l}J_{w}\left[\mathrm{~kg} \mathrm{~m}^{2}\right] \\
1.2\end{array}$ & $\begin{array}{l}B_{f} \\
10.4\end{array}$ & $\begin{array}{l}C_{f} \\
1.3\end{array}$ & $\begin{array}{l}B_{r} \\
21.4\end{array}$ & $\begin{array}{l}C_{r} \\
1.1\end{array}$ \\
\hline $\begin{array}{l}b_{b} \\
0.6\end{array}$ & $\begin{array}{l}\mu_{0} \\
1\end{array}$ & & & & \\
\hline \multicolumn{3}{|c|}{ SCENARIO PARAMETERS } & & & \\
\hline $\begin{array}{l}V(0)\left[\frac{m}{s}\right] \\
22\end{array}$ & $\begin{array}{l}X^{*}(0) \\
0\end{array}$ & $\begin{array}{l}X^{*}(4 \mathrm{~s})[\mathrm{m}] \\
70\end{array}$ & $\begin{array}{l}Y^{*}(0) \\
0\end{array}$ & $\begin{array}{l}\dot{Y}^{*}(0) \\
0\end{array}$ & $\begin{array}{l}\ddot{Y}^{*}(0) \\
0\end{array}$ \\
\hline $\begin{array}{l}\dddot{Y}^{*}(0) \\
0\end{array}$ & $\begin{array}{l}Y^{*}(2 \mathrm{~s})[\mathrm{m}] \\
\end{array}$ & $\begin{array}{l}Y^{*}(4 \mathrm{~s})[\mathrm{m}] \\
-1\end{array}$ & $\begin{array}{l}\dot{Y}^{*}(4 \mathrm{~s}) \\
0\end{array}$ & $\begin{array}{l}\ddot{Y}^{*}(4 \mathrm{~s}) \\
0\end{array}$ & $\begin{array}{l}\dddot{Y}^{*}(4 \mathrm{~s}) \\
0\end{array}$ \\
\hline
\end{tabular}

$$
\begin{aligned}
& \Lambda=u^{T} K(x) u+c(x) u+\lambda^{T}\left(a(x)+B(x) u-h_{P}^{(2)}(t)\right) \\
& \nabla \Lambda=0 \Rightarrow \\
& \left(\begin{array}{l}
u \\
\lambda
\end{array}\right)=\left(\begin{array}{cc}
2 K(x) & B^{T}(x) \\
B(x) & 0
\end{array}\right)^{-1}\left(\begin{array}{c}
-c^{T}(x) \\
-a(x)+h_{P}^{(2)}(t)
\end{array}\right) \\
& \text { V. RESULTS }
\end{aligned}
$$

\section{TABLE I}

For comparison of the optimal tracking controllers in Sec. III and the reactive tracking controller in Sec. IV, we use a double lane-change maneuver. The maneuver is also described as a benchmark problem in a previous work of the authors [6]. We specify the trajectory in earth-fixed coordinates $X$ and $Y$ by a polynomial of minimal degree, i.e., the number of parameters matches the number of constraints.

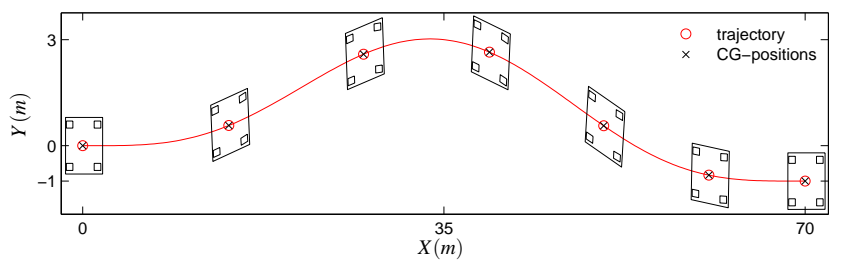

Fig. 1. Desired maneuver (trajectory) and open-loop simulation results (CG-positions, vehicle) for $\omega_{1 . .4}(t)$ and $\delta(t)$ values supplied by optimization without ESC.
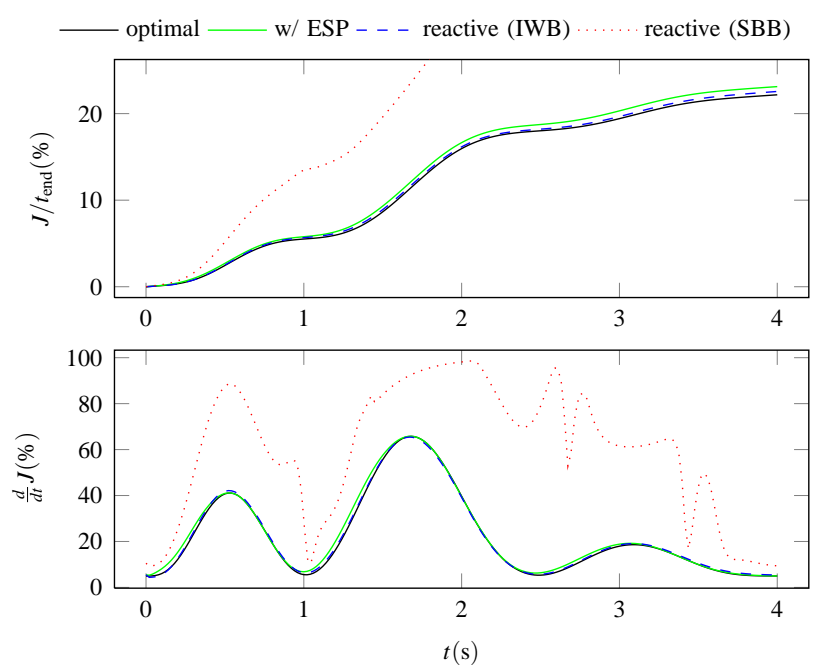

Fig. 2. Cost function value
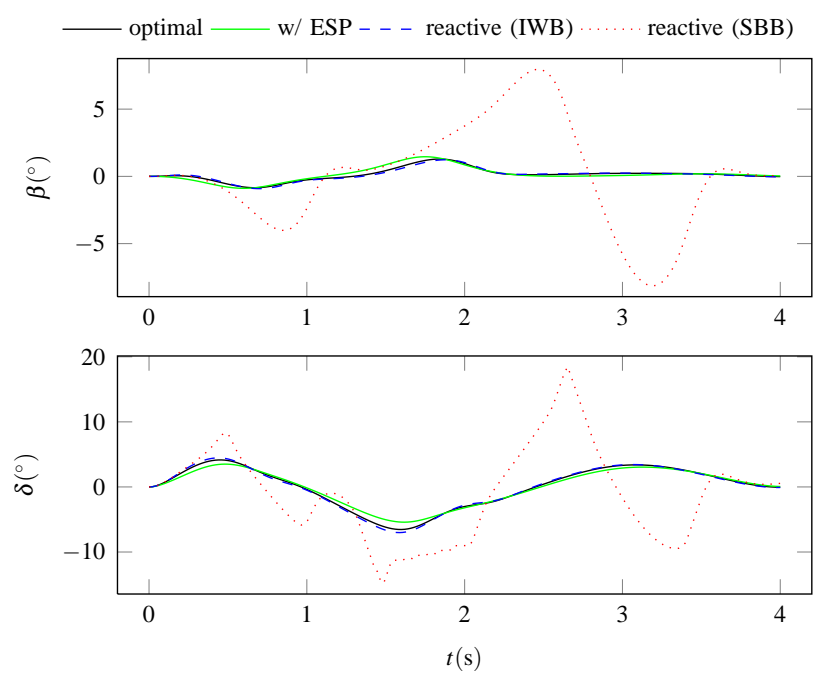

Fig. 3. Vehicle slip-angle and steering angle

The constraints are listed in Tab. I and the resulting trajectory is plotted in Fig. 1. The velocity profile along the path is given by constant deceleration from the initial velocity $V(0)$ so that the vehicle reaches the end of the given path after 4 seconds. The parameters of the vehicle model described in (1) can be found in Tab. I.

The four control concepts for comparison are: 1) optimal tracking, 2) optimal tracking with embedded yaw stabiliza- 

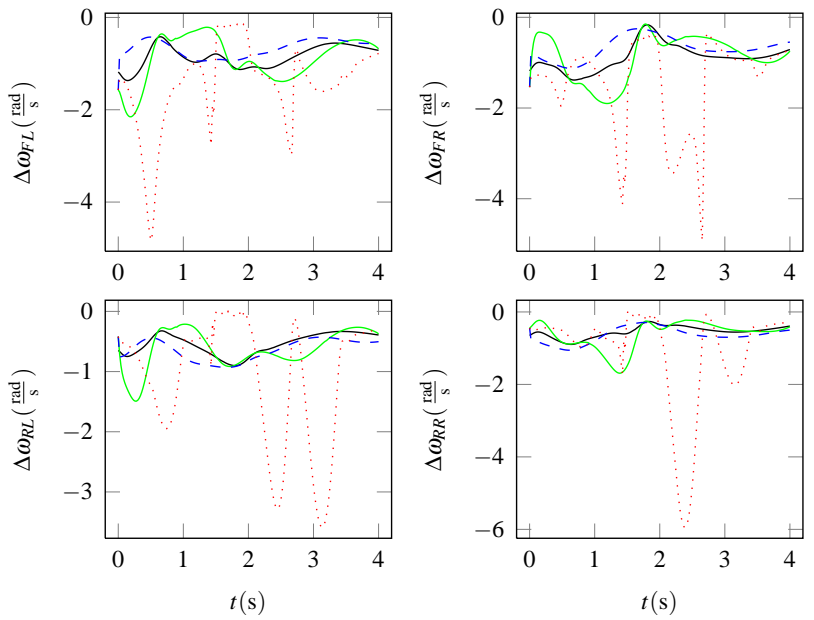

Fig. 4. Differential wheel-speeds

tion, 3) reactive tracking using individual brake torques, and 4) reactive tracking using the static brake balance. Since the comparison is set up for the case of exact tracking, which makes it possible to solely characterize yaw stabilization by the controlled yaw rate, only states comprising the zero dynamics differ:

- the slip angle relative to the trajectory $\beta$ (see Fig. 3) and its derivatives $\dot{\beta}$ and $\ddot{\beta}$,

- steering angle, Fig. 3

- wheel speed, Fig. 4

These variations cause different utilization of tire forces, which influences not only the ability to track the trajectory at low road friction values but also the friction reserves required to compensate tracking errors.

The resulting cost function of each maneuver is plotted in Fig. 2. It can be seen that the best performance is obtained by optimal tracking, followed by reactive tracking using individual brake torques, which performs even better than optimal tracking with embedded yaw stabilization. This shows that only using information of the current state can perform better compared to a global optimization enforcing embedded yaw stabilization. As expected, reactive tracking using the static brake balance performs worst. The plot clearly identifies an ordering for this benchmark problem since the cost functions retain their ordering for the complete time horizon. The cost function also provide a measure for the lost tire friction potential when yaw stabilization is activated.

\section{CONCLUSION}

We address the problem of comparing controllers for trajectory tracking in collision avoidance applications with and without embedded yaw stabilization. One of the main challenges for this comparison is that controllers for yaw stabilization differ from vehicle to vehicle. For that reason, an idealized yaw control is suggested that tracks a planned emergency maneuver without any tracking error. Even under this best case condition, optimal tracking control and reactive control using individual wheel torques perform better and thus possibly avoid collisions that are unavoidable by embedded yaw stabilization. Although existent in reality, the idealized yaw control does not consider an activation threshold. Upon deactivation of the yaw stabilization, the vehicle would most probably be forced to use a static brake balance, which performs even worse compared to yaw stabilization as presented in Sec. V. The results were derived under nominal conditions without either sensor noise, process disturbance or model mismatch. Therefore future work still has to investigate the robustness of the proposed methods and the performance under noisy conditions.

\section{REFERENCES}

[1] J. T. Betts. Practical methods for optimal control and estimation using nonlinear programming. Society for Industrial and Applied Mathematics, 2009.

[2] W. Cho, J. Choi, C. Kim, S. Choi, and K. Yi. Unified chassis control for the improvement of agility, maneuverability, and lateral stability. IEEE Transactions on Vehicular Technology, 61(3):1008-1020, 2012.

[3] T. Chung and K. Yi. Design and evaluation of side slip angle-based vehicle stability control scheme on a virtual test track. Control Systems Technology, IEEE Transactions on, 14(2):224-234, 2006.

[4] P. Falcone, H. Eric Tseng, F. Borrelli, J. Asgari, and D. Hrovat. Mpc-based yaw and lateral stabilisation via active front steering and braking. Vehicle System Dynamics, 46(S1):611-628, 2008.

[5] S. Fergani, O. Sename, and L. Dugard. Performances improvement through an $\mathrm{LPV} / H_{\infty}$ control coordination strategy involving braking, semi-active suspension and steering systems. In Proc. of the 51st IEEE Conference on Decision and Control, pages 4384-4389, 2012.

[6] D. Heß, M. Althoff, and T. Sattel. Comparison of trajectory tracking controllers for emergency situations. In Proc. of the 16th IEEE Conference on Intelligent Transportation Systems, 2013.

[7] K. Kritayakirana. Autonomous vehicle control at the limits of handling. $\mathrm{PhD}$ thesis, Stanford University, 2012.

[8] W. Langson and A. Alleyne. Multivariable bilinear vehicle control using steering and individual wheel torques. In Proc. of the American Control Conference, pages 1136-1140, 1997.

[9] O. Mokhiamar and M. Abe. How the four wheels should share forces in an optimum cooperative chassis control. Control Engineering Practice, 14(3):295-304, 2006.

[10] R. Orend. Integrierte Fahrdynamikregelung mit Einzelradaktorik - Ein Konzept zur Darstellung des fahrdynamischen Optimums. PhD thesis, Universität Erlangen-Nürnberg, 2006.

[11] S. Peters. Optimal Planning and Control for Hazard Avoidance of Front-Wheel Steered Ground Vehicles. PhD thesis, Massachusetts Institute of Technology, 2012.

[12] D. Piyabongkarn, J. Y. Lew, R. Rajamani, J. A. Grogg, and Q. Yuan. On the use of torque-biasing systems for electronic stability control: Limitations and possibilities. IEEE Transactions on Control Systems Technology, 15(3):581-589, 2007.

[13] R. Rajamani. Vehicle System Dynamics and Control. Springer, 2006.

[14] R. Rajamani and D. Piyabongkarn. New paradigms for the integration of yaw stability and rollover prevention functions in vehicle stability control. IEEE Transactions on Intelligent Transportation Systems, 14(1):249-261, 2013.

[15] B. Schofield and T. Hägglund. Optimal control allocation in vehicle dynamics control for rollover mitigation. In American Control Conference, pages 3231-3236, 2008.

[16] J. Tjønnås and T. A. Johansen. Stabilization of automotive vehicles using active steering and adaptive brake control allocation. IEEE Transactions on Control Systems Technology, 18(3):545-558, 2010.

[17] P. Tøndel and T. A. Johansen. Control allocation for yaw stabilization in automotive vehicles using multiparametric nonlinear programming. In Proc. of the American Control Conference, pages 453-458, 2005.

[18] M. Werling. Ein neues Konzept für die Trajektoriengenerierung und -stabilisierung in zeitkritischen Verkehrsszenarien. $\mathrm{PhD}$ thesis, Karlsruher Institut für Technologie, 2010.

[19] S. Yim, J. Choi, and K. Yi. Coordinated control of hybrid 4wd vehicles for enhanced maneuverability and lateral stability. IEEE Transactions on Vehicular Technology, 61(4):1946-1950, 2012. 